\title{
Genetic Analysis of Seed Yield and Its Contributing Traits and Pattern of Their Inheritance in Fieldpea (Pisum sativum L)
}

\author{
Manish Kumar ${ }^{1}$, M. S. Jeberson ${ }^{2 *}$, N. B. Singh ${ }^{3}$ and Ranjit Sharma ${ }^{3}$ \\ ${ }^{1}$ Plant Breeding and Genetics, BHU, Varanasi, India \\ ${ }^{2}$ Plant Breeding AICRP on MULLaRP, CAU, Imphal, India \\ ${ }^{3}$ Department of Genetics and Plant Breeding, COA, CAU, Imphal, India \\ *Corresponding author
}

\section{A B S T R A C T}

\section{Keywords}

Genetic analysis, Griffing's method II Model I, GCA,

SCA,

Field pea.

\section{Article Info}

Accepted:

04 May 2017

Available Online:

10 June 2017

\begin{abstract}
Aim of the study is analyse the inheritance of seed yield and its contributing traits through combining ability analysis in field pea for this seven genotypes as parents viz., Makyatmubi, Makuchabi, KPMR-851, Prakash, Pant P-217, Rachna and VL-5 in diallel without reciprocals during Rabi 2013-14 were selected. The genetic analysis was carried out following Griffing's Method II with Model I (1956) for twelve quantitative characters. The ANOVA for combining ability revealed highly significant differences among crosses for all the characters studied. The $\sigma 2 \mathrm{GCA} / \sigma 2 \mathrm{SCA}$ ratio was shown to be less than unity for most of the character indicating the predominant role of non-additive gene action in the inheritance of those traits. However, for remaining traits days to first flowering, number of nods to first flowering, number of seeds per pod and 100 seed weight, the ratio was found to be more equal to unity indicating the importance of both additive and non-additive gene action in the expression of these gene. In case of GCA effects, Makyatmubi and Makuchabi were identified as the most promising parents for involving in hybridization programme. On the basis of SCA effects, two crosses viz., Makyatmubi x KPMR-851 and Makuchabi x VL-58 were identified as the most promising crosses for improvement of seed yield per plant viz., number of pods/plant, number of seeds/pod, etc. These crosses showing highly significant SCA effects for seed yield per plant also exhibited high per se performance and moreover both the parents involved either as good general combiner or at least one good combiner for seed yield per plant. The manifestation of heterosis for seed yield was evidenced by superiority of hybrids ranging from 42.28 to $192.48 \%$ in 19 crosses over standard check variety Rachna. Overall on the basis of results of mean performance, including GCA and SCA effects and standard heterosis, three crosses viz., Makyatmubi x KPMR-851, Makuchabi x VL-58 and Makuchabi x Prakash were identified as the most promising cross combinations for improvement of seed yield and its
\end{abstract}

\section{Introduction}

Pulses (grain legumes) are important group of crops which are grown not only for protein but also have considerable amount of carbohydrates, minerals and vitamin B complex. Among pulses, pea (Pisum sativum L.), also known as field pea and garden pea in English, and Matar in Hindi is one of the important rabi (winter) crops grown in the world and India. Pea $(2 n=2 x=14)$ belongs to the family Leguminoseae and genus Pisum. It is an annual herbaceous, self-pollinated crop. Field pea is one of the important pulse crops in India, grown in an area of 0.68 million hectares producing $0.62 \mathrm{MT}$ of grain. The 
average national productivity of fieldpea is $911 \mathrm{~kg} / \mathrm{ha}$ (Anonymous, 2013). It is consumed as both green immature seeds as well as dry seeds. Like other pulses, field pea is used along with cereals such as rice, wheat, maize or millets so as to balance the level of essential amino acids, as pulses are deficient in methionine and rich in lysine, while cereals are rich in methionine and deficient in lysine (Srivastava and Ali, 2004). Field pea is an important pulse crop in Manipur during rabi season. In Manipur, the productivity of pea is lower $(767 \mathrm{~kg} / \mathrm{ha})$ as compared to national level $(911 \mathrm{~kg} / \mathrm{ha})$. One of the major constraint in increasing the area and productivity of pea in Manipur is the lack of high yielding varieties which are suitable for cultivation under varied agro-climatic conditions of the state. Development of high yielding genotypes depends on the selection of parents. Combining ability analysis provides a means of selection of parents. Hence, the present investigation was carried out to help selection of parents.

\section{Materials and Methods}

The present investigation was conducted at the research field of the Department of Plant Breeding and Genetics, College Of Agriculture, Central Agricultural University, Imphal. The experiment consisted of evaluations of $21 \mathrm{~F} 1 \mathrm{~s}$ obtained by crossing seven parents in a half diallel fashion. The parents, viz., Makyatmubi, Makuchabi, KPMR-851, Prakash, Pant P-217, Rachna and VL-5 were randomly chosen from a collection maintained at CAU, Imphal. The seven parents along with $21 \mathrm{~F} 1 \mathrm{~s}$ are evaluated during rabi 2014-15 in a RBD with three replications. Each F1 parent was sown in a plot. Each plot consisted of a single row spaced $30 \mathrm{~cm}$ with a with length of $4 \mathrm{~m}$. The plant to plant distance was $10 \mathrm{~cm}$ and appropriate agronomical practices were followed to raise a good crop.
Observations were recorded on five individual plants taken at random (excluding border plants) from each genotype for days to first flowering, number of nodes to first flowering, days to $50 \%$ flowering, days to maturity, plant height $(\mathrm{cm})$, number of pods/plant, pod length (cm), number of seeds/pod, seed yield/plant (g), biological yield/plant (g), 100 seed weight $(\mathrm{g})$, harvest index (HI) (\%). The analysis of variance was done according to the method given by Griffings (1956). Heterosis was worked out over better parent and its significance was determined by $t$ test as suggested by Rai and Rai (2006).

\section{Results and Discussion}

The analysis of variance revealed significant differences among the parents and F1s for all the characters (Table 1). These findings showed that enough genetic variability available in the materials studied. Bisht and Singh (2011), Brar et al., (2012) and Esposito et al., (2013) had also observed significant differences among the genotypes for different characters viz., days to first flowering, nodes to first flowering, days to $50 \%$ flowering, days to maturity, plant height, number of pods/plant, pod length, number of seeds/pod, seed yield/plant, biological yield/plant, 100 seed weight and harvest index.

The analysis of variance for combining ability revealed that mean squares due to both general combining ability and specific combining ability were highly significant for all the traits investigated (Table 1). This indicated the importance of both additive and non-additive gene action for the expression of almost all the characters. However, except the few characters viz., days to first flowering, number of nodes to first flowering, number of seeds/plant and 100 seed weight, the ratio of general combining ability and specific combining ability $(\sigma 2 \mathrm{~g} / \sigma 2 \mathrm{~s})$ was shown to be less than unity for all other characters 
indicating the predominant role of nonadditive gene effect for the expression of these traits. These findings are in corroborating with the findings of Singh et al., (1994) and Sharma et al., (2003). The per se performance of the parents was good indicator for their general combining effects (Table 2). The parent Makyatmubi recorded significantly positive gca effects for nodes to first flowering, pod length, seed yield/plant, biological yield/plant and 100 seed weight.

Makuchabi was found to be good general combiners for nodes to first flowering, pod length, number of seeds/pod, seed yield/plant and harvest index. The similar results were reported by Pant and Bajpai (1993), Pandey et al., (1996) and Kumar and Jain (2002). It is evident from table 2 that the significant gca effects for seed yield in positive direction resulted from similar gca effects of some yield components indicating that the combining ability of seed yield was influenced by the combined effects of its components. Therefore, simultaneous improvement in important yield components and associated trait along with seed yield may be better approach for raising yield potential in pea. The estimates of specific combining ability effects of 21 F1 crosses for 12 characters under study are presented in table 3.

The negative and significant specific combining ability effects were found in the cross KPMR-851 x Prakash for days to first flowering, Prakash x Pant P-217 for days to $50 \%$ flowering, Prakash $\mathrm{x}$ Rachna and KPMR-851 x Rachna for days to maturity, Makuchabi x Pant P-217 for plant height. So these crosses can be utilized for evolving early flowering, maturing and dwarf plants. Makuchabi x VL-58, Makyatmubi x KPMR851, Pant P-217 x Rachna, Makuchabi X Prakash and Prakash x VL-58 for number of pods/plant, Makyatmubi X VL-58 and
Makyatmubi x Prakash for 100 seed weight exhibited highly significant and positive specific combining ability effects. Ranjan et al., (2005) and Zaman and Hazarika (2005) were also obtained similar results while studying combining ability in field pea.

The range of heterosis for different characters over standard check were from 42.28 to 192.75 percent for seed yield, -13.43 to 2.49 percent for days to first flowering, 2.56 to 25.64 percent for number of nodes to first flowering, -11.16 to 1.86 percent for days to $50 \%$ flowering, -4.44 to -0.59 percent for days to maturity, -2.03 to 24.63 percent for plant height, 4.35 to 69.57 percent for pods/plant, -3.79 to 22.05 percent for pod length, -6.25 to 25.00 percent for seeds/pod, 0.02 to 59.15 percent for 100 seed weight, 25.96 to 135.52 percent for biological yield/plant and 11.98 to 43.29 percent for harvest index respectively.

Similar results were reported by Pant and Bajpai (1991) and Sharma et al., (1998) in field pea. The list of best crosses for different characters showing heterosis over standard check (SC) is given in table 4. Most of the hybrids showed negative heterosis for days to 50 percent flowering and days to maturity indicating that they had the tendency to flower and mature early. High magnitudes of heterosis were observed for 100 seed weight. This finding is corroboration with the results of Bora (2009). Plant breeders can give emphasis on yield contributing characters for the improvement of seed yield in field pea.

From the table 5, it is revealed that most of the good specific cross combinations for different characters involved parents of low $\mathrm{x}$ low, low $\mathrm{x}$ average, average $\mathrm{x}$ average, average $\mathrm{x}$ high and high $\mathrm{x}$ high general combining ability. The classification of low, average and high of the parents was done based on their seed yield. 
Int.J.Curr.Microbiol.App.Sci (2017) 6(6): 172-181

Table.1 Analysis of variance for combining ability for different characters in 7- parent half-diallel of field pea

\begin{tabular}{|c|c|c|c|c|c|c|c|}
\hline \multirow[t]{2}{*}{ Source of variation } & \multirow[t]{2}{*}{ d.f. } & \multicolumn{6}{|c|}{ Mean sum of squares } \\
\hline & & $\begin{array}{c}\text { Days to first } \\
\text { flowering }\end{array}$ & $\begin{array}{c}\begin{array}{c}\text { Nodes to first } \\
\text { flowering }\end{array} \\
\end{array}$ & $\begin{array}{c}\text { Days to 50\% } \\
\text { flowering }\end{array}$ & Days to maturity & $\begin{array}{c}\begin{array}{c}\text { Plant height } \\
(\mathrm{cm})\end{array} \\
\end{array}$ & $\begin{array}{l}\text { Number of } \\
\text { pods/plant }\end{array}$ \\
\hline GCA & 6 & $20.728 * *$ & $3.442 * *$ & $13.350 * *$ & $2.888^{*}$ & $228.780 * *$ & $3.008 * *$ \\
\hline SCA & 21 & 4.939 & 0.432 & $6.161 * *$ & 2.371 & 238.886 & $3.347 * *$ \\
\hline Error & 54 & 3.162 & 0.338 & 2.645 & 1.087 & 22.189 & 0.866 \\
\hline$\sigma^{2}$ gca & & 1.952 & 0.345 & 1.189 & 0.2 & 22.955 & 0.238 \\
\hline$\sigma^{2} \mathrm{sca}$ & & 1.778 & 0.093 & 3.515 & 1.285 & 216.697 & 2.48 \\
\hline$\sigma^{2}$ gca $/ \sigma^{2}$ sca & & 1.098 & 3.695 & 0.338 & 0.156 & 0.106 & 0.096 \\
\hline
\end{tabular}

\begin{tabular}{|c|c|c|c|c|c|c|c|}
\hline \multirow[t]{2}{*}{ Source of variation } & \multirow[t]{2}{*}{ d.f. } & \multicolumn{6}{|c|}{ Mean sum of squares } \\
\hline & & Pod length $(\mathrm{cm})$ & $\begin{array}{l}\text { Number of } \\
\text { seeds/pod }\end{array}$ & $\begin{array}{c}\text { seed yield/plant } \\
\text { (g) }\end{array}$ & $\begin{array}{c}\text { biological } \\
\text { yield/plant (g) }\end{array}$ & $\begin{array}{c}100 \text { seed } \\
\text { weight }(\mathrm{g})\end{array}$ & $\begin{array}{c}\text { Harvest index } \\
(\%)\end{array}$ \\
\hline GCA & 6 & $0.456 * *$ & $0.904 * *$ & $8.293 * *$ & $34.246^{* *}$ & $25.009 * *$ & $26.847 * *$ \\
\hline SCA & 21 & 0.092 & 0.117 & $7.962 * *$ & $31.635 * *$ & $1.996 * *$ & $12.508 * *$ \\
\hline Error & 54 & 0.041 & 0.134 & 1.352 & 6.523 & 0.757 & 3.517 \\
\hline$\sigma^{2}$ gca & & 0.046 & 0.086 & 0.771 & 3.08 & 2.695 & 2.592 \\
\hline$\sigma^{2}$ sca & & 0.051 & 0.017 & 6.61 & 25.112 & 1.24 & 8.992 \\
\hline$\sigma^{2}$ gca $/ \sigma^{2}$ sca & & 0.903 & 5.018 & 0.117 & 0.123 & 2.174 & 0.288 \\
\hline
\end{tabular}

$*$,** Significant at $5 \%$ and $1 \%$ levels, respectively 
Table.2 Estimates of general combining ability effects for days to first flowering, nodes to first flowering, and days to 50\% flowering, days to maturity, plant height and Number of pods/plant in a half-diallel crosses of field pea

\begin{tabular}{|c|c|c|c|c|c|c|c|c|c|c|c|c|}
\hline \multirow[b]{2}{*}{ Parent } & \multicolumn{2}{|c|}{ Days to first flowering } & \multicolumn{2}{|c|}{ Number of nodes to first flowering } & \multicolumn{2}{|c|}{ Days to $50 \%$ flowering } & \multicolumn{2}{|c|}{ Days to maturity } & \multicolumn{2}{|c|}{ Plant Height $(\mathrm{cm})$} & \multicolumn{2}{|l|}{ Pods/plant } \\
\hline & GCA effect & Mean & GCA effect & Mean & GCA effect & Mean & GCA effect & Mean & GCA effect & Mean & GCA effect & Mean \\
\hline 1.Makyatmubi & 0.905 & 63.00 & $0.561 *$ & 15.33 & $1.037 *$ & 69.00 & 0.317 & 110.33 & 0.089 & 111.27 & -0.111 & 6.67 \\
\hline 2. Makuchabi & 0.349 & 63.67 & $0.635^{*}$ & 15.67 & 0.407 & 69.33 & -0.016 & 110.67 & $-3.193 *$ & 105.53 & 0.333 & 7.33 \\
\hline 3. KPMR-851 & $-1.947 *$ & 62.33 & -0.069 & 15.33 & $-1.889 *$ & 67.00 & $-0.831 *$ & 110.00 & 0.156 & 109.27 & $1.148^{*}$ & 8.67 \\
\hline 4. Prakash & $1.423^{*}$ & 70.33 & $0.561 *$ & 15.33 & $1.074 *$ & 74.67 & 0.577 & 114.00 & $-4.844 *$ & 58.73 & $-0.593 *$ & 6.00 \\
\hline 5. Pant P-217 & $-1.614 *$ & 61.67 & -0.143 & 14.33 & $-1.259 *$ & 68.67 & -0.608 & 110.00 & $10.704 *$ & 132.07 & -0.259 & 7.67 \\
\hline 6. Rachna & $1.868 *$ & 67.00 & $-0.624 *$ & 13.00 & $1.074 *$ & 71.67 & $0.651 *$ & 112.67 & -1.556 & 111.53 & -0.333 & 7.67 \\
\hline 7. VL-58 & -0.984 & 61.67 & $-0.921 *$ & 13.67 & -0.444 & 70.67 & -0.090 & 112.33 & -1.356 & 104.33 & -0.185 & 7.33 \\
\hline Mean & & 64.24 & & 14.67 & & 70.14 & & 111.43 & & 104.68 & & 7.33 \\
\hline SE(gi) & 0.549 & & 0.179 & & 0.501 & & 0.322 & & 1.453 & & 0.287 & \\
\hline $\mathrm{SE}$ (gi-gj) & & & 0.274 & & 0.767 & & 0.491 & & 2.22 & & 0.439 & \\
\hline
\end{tabular}

\begin{tabular}{|c|c|c|c|c|c|c|c|c|c|c|c|c|}
\hline \multirow[b]{2}{*}{ Parent } & \multicolumn{2}{|c|}{ Pod length $(\mathrm{cm})$} & \multicolumn{2}{|c|}{ Number of seeds/pod } & \multicolumn{2}{|c|}{ Seed yield/plant (g) } & \multicolumn{2}{|c|}{ Biological yield/plant (g) } & \multicolumn{2}{|c|}{100 seed weight(g) } & \multicolumn{2}{|c|}{ Harvest index $(\%)$} \\
\hline & GCA effect & Mean & GCA effect & Mean & GCA effect & Mean & GCA effect & Mean & GCA effect & Mean & GCA effect & Mean \\
\hline 1.Makyatmubi & $0.348 *$ & 7.27 & -0.212 & 5.00 & $1.269 *$ & 8.70 & $3.768 *$ & 21.47 & $3.039 *$ & 23.51 & -0.519 & 40.43 \\
\hline 2. Makuchabi & $0.286^{*}$ & 6.82 & $0.455^{*}$ & 6.33 & $0.876^{*}$ & 6.60 & 0.648 & 16.46 & 0.490 & 18.43 & 1.842 & 40.11 \\
\hline 3. KPMR-851 & -0.110 & 6.37 & $-0.249 *$ & 5.67 & 0.082 & 7.73 & 0.223 & 20.96 & $-0.608^{*}$ & 18.46 & 0.333 & 36.76 \\
\hline 4. Prakash & -0.075 & 6.37 & $-0.323 *$ & 5.00 & -0.149 & 6.77 & -1.022 & 15.78 & $1.074 *$ & 20.93 & 1.283 & 43.17 \\
\hline 5. Pant P-217 & -0.047 & 6.61 & $0.418^{*}$ & 6.33 & 0.199 & 7.70 & -0.345 & 18.14 & $-1.340 *$ & 17.42 & 1.662 & 42.42 \\
\hline 6. Rachna & $-0.223^{*}$ & 5.99 & -0.101 & 5.33 & $-1.634 *$ & 5.29 & $-2.542 *$ & 15.72 & $-1.747 *$ & 15.76 & $-2.961 *$ & 33.44 \\
\hline 7. VL-58 & $-0.179 *$ & 6.49 & 0.011 & 6.00 & -0.643 & 6.21 & -0.730 & 18.48 & $-0.909 *$ & 18.25 & -0.974 & 33.50 \\
\hline Mean & & 6.56 & & 5.67 & & 7.00 & & 18.14 & & 18.97 & & 38.55 \\
\hline SE(gi) & 0.062 & & 0.113 & & 0.359 & & 0.788 & & 0.268 & & 0.578 & \\
\hline SE(gi-gj) & 0.096 & & 0.173 & & 0.548 & & 1.204 & & & & 0.884 & \\
\hline
\end{tabular}

*,** Significant at $5 \%$ and $1 \%$ levels, respectively 


\section{Int.J.Curr.Microbiol.App.Sci (2017) 6(6): 172-181}

Table.3 Estimates of specific combining ability effects for days to first flowering, nodes to first flowering, and days to 50\% flowering, days to maturity, plant height and Number of pods/plant in a half-diallel crosses of field pea

\begin{tabular}{|c|c|c|c|c|c|c|c|c|c|c|c|c|}
\hline \multirow[b]{2}{*}{ Parent } & \multicolumn{2}{|c|}{ Days to first flowering } & \multicolumn{2}{|c|}{$\begin{array}{l}\text { Number of nodes to first } \\
\text { flowering }\end{array}$} & \multicolumn{2}{|c|}{ Days to $50 \%$ flowering } & \multicolumn{2}{|c|}{ Days to maturity } & \multicolumn{2}{|c|}{ Plant Height $(\mathrm{cm})$} & \multicolumn{2}{|l|}{ Pods/plant } \\
\hline & SCA effect & Mean & SCA effect & Mean & SCA effect & Mean & $\begin{array}{l}\text { SCA } \\
\text { effect }\end{array}$ & Mean & SCA effect & Mean & $\begin{array}{l}\text { SCA } \\
\text { effect }\end{array}$ & Mean \\
\hline Makyatmubi x Makuchabi & 0.556 & 64.67 & 0.185 & 16.33 & -0.861 & 68.33 & -0.694 & 109.33 & 2.679 & 118.07 & 0.611 & 10.00 \\
\hline Makyatmubi x KPMR851 & -1.148 & 60.67 & 0.556 & 16.00 & 0.102 & 67.00 & -0.213 & 109.00 & 1.931 & 120.67 & $2.796 * *$ & 13.00 \\
\hline Makyatmubi X Prakash & 1.815 & 67.00 & 0.259 & 16.33 & 1.139 & 71.00 & 0.380 & 111.00 & $15.064 * *$ & 128.80 & 0.537 & 9.00 \\
\hline Makyatmubi x Pant P 217 & 0.519 & 62.67 & 0.296 & 15.67 & -0.861 & 66.67 & -0.435 & 109.00 & 5.182 & 134.47 & 1.204 & 10.00 \\
\hline Makyatmubi x Rachna & 3.037 & 68.67 & 0.111 & 15.00 & $3.139 *$ & 73.00 & 1.306 & 112.00 & -4.358 & 112.67 & -0.722 & 8.00 \\
\hline Makyatmubi x VL 58 & -1.444 & 61.33 & 0.074 & 14.67 & -1.009 & 67.33 & -0.287 & 109.67 & -5.692 & 111.53 & 0.13 & 9.00 \\
\hline Makuchabi x KPMR851 & -1.259 & 60.00 & 0.815 & 16.33 & -0.602 & 65.67 & 0.120 & 109.00 & 1.012 & 116.47 & 0.019 & 10.67 \\
\hline Makuchabi X Prakash & -1.963 & 62.67 & -0.148 & 16.00 & -2.231 & 67.00 & -1.287 & 109.00 & $9.945^{*}$ & 120.40 & 1.426 & 10.33 \\
\hline Makuchabi x Pant P 217 & 1.407 & 63.00 & 0.556 & 16.00 & 0.769 & 67.67 & 0.898 & 110.00 & $-10.036^{*}$ & 115.97 & -0.907 & 8.33 \\
\hline Makuchabi x Rachna & 1.259 & 66.33 & 0.704 & 15.67 & 2.102 & 71.33 & 0.639 & 111.00 & 4.656 & 118.40 & 0.167 & 9.33 \\
\hline Makuchabi x VL 58 & -0.222 & 62.00 & -1.000 & 13.67 & -0.713 & 67.00 & -1.620 & 108.00 & 0.890 & 118.83 & $3.685 * *$ & 13.00 \\
\hline KPMR851 X Prakash & $-4.000 *$ & 58.33 & -0.444 & 15.00 & -1.935 & 65.00 & -1.139 & 108.33 & $18.331 * *$ & 132.13 & 0.278 & 10.00 \\
\hline KPMR851 x Pant P 217 & -1.296 & 58.00 & -0.407 & 14.33 & 0.398 & 65.00 & -0.287 & 108.00 & 1.049 & 130.40 & $2.611 * *$ & 12.67 \\
\hline KPMR851 x Rachna & -0.444 & 62.33 & -0.926 & 13.33 & -2.269 & 64.67 & $-1.880 *$ & 107.67 & -2.758 & 114.33 & $1.685 *$ & 11.67 \\
\hline KPMR851 x VL 58 & 1.407 & 61.33 & -0.63 & 13.33 & -1.75 & 63.67 & -0.472 & 108.33 & -0.492 & 116.80 & $-1.796^{*}$ & 8.33 \\
\hline Prakash x Pant P 217 & -2.667 & 60.00 & 0.63 & 16.00 & $-3.565^{*}$ & 64.00 & -1.361 & 108.33 & $14.649 * *$ & 139.00 & -0.315 & 8.00 \\
\hline Prakash x Rachna & -2.815 & 63.33 & 0.778 & 15.67 & -2.565 & 67.33 & $-1.954 *$ & 109.00 & $18.375 * *$ & 130.47 & 0.426 & 8.67 \\
\hline Prakash x VL 58 & 0.37 & 63.67 & 0.407 & 15.00 & -0.380 & 68.00 & -0.880 & 109.33 & $23.775 * *$ & 136.07 & 1.611 & 10.00 \\
\hline Pant P 217 x Rachna & -1.111 & 62.00 & 0.148 & 14.33 & -1.898 & 65.67 & -0.435 & 109.33 & -1.906 & 125.73 & 0.093 & 8.67 \\
\hline Pant P 217 x VL 58 & -0.926 & 59.33 & -0.556 & 13.33 & -1.713 & 64.33 & -1.361 & 107.67 & 6.727 & 134.57 & -0.722 & 8.00 \\
\hline Rachna x VL 58 & -0.741 & 63.00 & 0.593 & 14.00 & -2.046 & 66.33 & -0.954 & 109.33 & -6.314 & 109.27 & 0.019 & 8.67 \\
\hline Mean & & 59.4 & & 15.05 & & 67.00 & & 109.16 & & 123.10 & & 9.78 \\
\hline SE(si) & 1.596 & & 0.521 & & 1.459 & & 0.935 & & 4.227 & & 0.835 & \\
\hline SE(si-sj) & 1.874 & & 0.613 & & 1.714 & & 1.099 & & 4.965 & & 0.981 & \\
\hline
\end{tabular}




\begin{tabular}{|c|c|c|c|c|c|c|c|c|c|c|c|c|}
\hline \multirow[b]{2}{*}{ Parent } & \multicolumn{2}{|c|}{ Pod length (cm) } & \multicolumn{2}{|c|}{ Number of seeds/pod } & \multicolumn{2}{|c|}{ Seed yield/plant (g) } & \multicolumn{2}{|c|}{ Biological yield/plant } & \multicolumn{2}{|c|}{100 seed weight $(\mathrm{g})$} & \multicolumn{2}{|c|}{ Harvest index (\%) } \\
\hline & SCA effect & Mean & SCA effect & Mean & SCA effect & Mean & SCA effect & Mean & SCA effect & Mean & SCA effect & Mean \\
\hline Makyatmubi x Makuchabi & -0.227 & 7.04 & 0.269 & 6.33 & 1.025 & 13.80 & 0.99 & 30.52 & 1.376 & 25.08 & 1.462 & 45.12 \\
\hline Makyatmubi x KPMR851 & 0.258 & 7.13 & -0.028 & 5.33 & $3.505^{* *}$ & 15.49 & $7.911^{* *}$ & 37.02 & 0.010 & 22.61 & 0.030 & 41.52 \\
\hline Makyatmubi X Prakash & $0.403^{*}$ & 7.31 & 0.38 & 5.67 & 0.269 & 12.02 & 4.433 & 32.29 & $1.619^{*}$ & 25.90 & $-5.656^{* * *}$ & 37.45 \\
\hline Makyatmubi x Pant P 217 & 0.341 & 7.27 & 0.306 & 6.33 & 1.676 & 13.77 & 3.189 & 31.73 & 0.212 & 22.08 & -0.175 & 43.31 \\
\hline Makyatmubi x Rachna & $-0.429 *$ & 6.33 & -0.176 & 5.33 & 1.931 & 12.20 & $5.193 *$ & 31.53 & -0.254 & 21.21 & 0.058 & 38.92 \\
\hline Makyatmubi x VL 58 & 0.240 & 6.56 & 0.046 & 5.67 & 0.531 & 11.79 & 0.651 & 28.80 & $2.524^{* *}$ & 24.83 & $6.021 * *$ & 46.87 \\
\hline Makuchabi x KPMR851 & -0.053 & 6.75 & -0.028 & 6.00 & 1.829 & 13.42 & 1.892 & 27.88 & -0.337 & 19.72 & $3.970 *$ & 47.81 \\
\hline Makuchabi X Prakash & 0.273 & 7.11 & 0.38 & 6.33 & $2.553^{*}$ & 13.91 & 4.277 & 29.02 & 1.415 & 23.15 & 454 & 47.92 \\
\hline Makuchabi x Pant P 217 & 0.054 & 6.92 & -0.361 & 6.33 & 0.896 & 12.60 & 1.640 & 27.06 & 1.091 & 20.41 & 1.025 & 46.87 \\
\hline Makuchabi x Rachna & 0.267 & 6.96 & 0.157 & 6.33 & 0.751 & 10.62 & 2.007 & 25.23 & 0.949 & 19.86 & 0.958 & 42.18 \\
\hline Makuchabi x VL 58 & $0.450 *$ & 7.19 & 0.38 & 6.67 & $4.501^{* *}$ & 15.36 & $9.095^{* *}$ & 34.13 & 0.957 & 20.71 & 1.961 & 45.17 \\
\hline KPMR851 X Prakash & -0.012 & 6.43 & -0.25 & 5.00 & 1.170 & 11.73 & 1.119 & 25.43 & 0.433 & 21.07 & 2.905 & 46.19 \\
\hline KPMR851 x Pant P 217 & -0.300 & 6.17 & -0.324 & 5.67 & 0.523 & 11.43 & 0.805 & 25.80 & 0.193 & 18.42 & 0.803 & 44.47 \\
\hline KPMR851 x Rachna & 0.189 & 6.49 & 0.194 & 5.67 & -0.585 & 8.49 & -0.798 & 22.00 & 0.190 & 18.01 & -0.11 & 38.93 \\
\hline KPMR851 x VL 58 & -0.008 & 6.33 & -0.25 & 5.33 & -0.312 & 9.76 & -1.734 & 22.87 & 0.495 & 19.15 & 2.233 & 43.27 \\
\hline Prakash x Pant P 217 & 0.065 & 6.57 & 0.417 & 6.33 & 0.72 & 11.40 & 1.513 & 25.26 & 0.085 & 19.99 & -0.17 & 45.11 \\
\hline Prakash x Rachna & 0.101 & 6.43 & -0.065 & 5.33 & 0.256 & 9.10 & -0.482 & 21.07 & 0.532 & 20.03 & 2.37 & 43.03 \\
\hline Prakash x VL 58 & $-0.616 * *$ & 5.76 & -0.509 & 5.00 & $2.152 *$ & 11.99 & 3.718 & 27.08 & -1.300 & 19.04 & 1.57 & 44.22 \\
\hline Pant P 217 x Rachna & -0.008 & 6.35 & 0.528 & 6.67 & $2.619 *$ & 11.81 & $4.750 *$ & 26.98 & 0.725 & 17.81 & 2.838 & 43.88 \\
\hline Pant P 217 x VL 58 & -0.305 & 6.10 & 0.083 & 6.33 & 0.221 & 10.41 & 0.664 & 24.70 & $-2.170^{*}$ & 15.75 & 2.157 & 45.18 \\
\hline Rachna x VL 58 & 0.278 & 6.51 & -0.065 & 5.67 & -0.826 & 7.53 & -2.045 & 19.80 & $\begin{array}{r}-0.299 \\
\end{array}$ & 17.22 & -0.163 & 38.24 \\
\hline Mean & & 6.65 & & 5.87 & & 11.84 & & 27.44 & & 20.57 & & 43.60 \\
\hline SE(sij) & 0.182 & & 0.328 & & 1.043 & & 2.292 & & 0.781 & & 1.683 & \\
\hline SE(sii-gjj) & 0.214 & & 0.386 & & 1.226 & & 2.692 & & 0.917 & & 1.978 & \\
\hline
\end{tabular}

*, ** Significant at 5\% and $1 \%$ levels, respectively 
Table.4 Top crosses showing significant desirable sca effects, their gca effects and mean per se performance

\begin{tabular}{|c|c|c|c|}
\hline Character & Sca effects & Gca effects & Per se performance \\
\hline Days to first flowering & KPMR-851 X Prakash(-4) & $\mathrm{H} \mathrm{X} \mathrm{L}$ & KPMR-851 X Prakash(58.33) \\
\hline \multicolumn{4}{|l|}{ Nodes to first flowering } \\
\hline Days to $50 \%$ flowering & Prakash X Pant P-217(-3.56) & L X H & Prakash X Pant P-217(64) \\
\hline \multirow[t]{2}{*}{ Days to maturity } & Prakash X Rachna(-1.95) & A X L & Prakash X Rachna(109) \\
\hline & KPMR-851 X Rachna(-1.88) & H X L & KPMR-851 X Rachna(107.67) \\
\hline Plant height (cm) & Makuchabi X Pant P-217(-10.03) & L X H & Makuchabi X Pant P-217(115.97) \\
\hline \multirow[t]{4}{*}{ Number of pods/plant } & Makuchabi X VL-58(3.68) & $\mathrm{A} X \mathrm{~A}$ & Makuchabi X VL-58(13) \\
\hline & Makyatmubi X KPMR-851(2.79) & A X H & Makyatmubi X KPMR-851(13) \\
\hline & KPMR-851 X Pant P-217(2.61) & HX A & KPMR-851 X Pant P-217(12.67) \\
\hline & KPMR-851 X Rachna(1.68) & HX A & KPMR-851 X Rachna(11.67) \\
\hline \multirow[t]{2}{*}{ Pod length $(\mathrm{cm})$} & Makuchabi X VL-58(0.45) & $\mathrm{HXL}$ & Makyatmubi X Prakash(7.31) \\
\hline & Makyatmubi X Prakash(0.403) & HX A & Makuchabi X VL-58(7.19) \\
\hline \multicolumn{4}{|l|}{ Number of seeds/pod } \\
\hline \multirow[t]{5}{*}{ Seed yield/plant (g) } & Makuchabi X VL-58(4.5) & HX A & Makyatmubi X KPMR-851(15.49) \\
\hline & Makyatmubi X KPMR-851(3.5) & H X A & Makuchabi X VL-58(15.36) \\
\hline & Pant P-217 X Rachna(2.62) & $\mathrm{AXL}$ & Makuchabi X Prakash(13.91) \\
\hline & Makuchabi X Prakash(2.55) & $\mathrm{HX} \mathrm{A}$ & Prakash X VL-58(11.99) \\
\hline & Prakash X VL-58(2.12) & $\mathrm{A} X \mathrm{~A}$ & Pant P-217 X Rachna(11.81) \\
\hline \multirow[t]{4}{*}{ Biological yield/plant (g) } & Makuchabi X VL-58(9.09) & A X A & Makyatmubi XKPMR-851(37.02) \\
\hline & Makyatmubi X KPMR-851(7.91) & $\mathrm{HX} \mathrm{A}$ & Makuchabi X VL-58(34.13) \\
\hline & Makyatmubi X Rachna(5.19) & $\mathrm{HX} \mathrm{L}$ & Makyatmubi X Rachna(31.53) \\
\hline & Pant P-217 X Rachna(4.75) & A X L & Pant P-217 X Rachna(26.98) \\
\hline \multirow[t]{2}{*}{100 seed weight (g) } & Makyatmubi X VL-58(2.52) & H X L & Makyatmubi X Prakash(25.9)) \\
\hline & Makyatmubi X Prakash(1.62) & $\mathrm{HX} \mathrm{H}$ & Makyatmubi X VL-58(24.83) \\
\hline \multirow[t]{2}{*}{ Harvest index $(\%)$} & Makyatmubi X VL-58(6.02) & A X A & Makuchabi X KPMR-851(47.81) \\
\hline & Makuchabi X KPMR-851(3.97) & H X A & Makyatmubi X VL-58(46.87) \\
\hline
\end{tabular}


Table.5 Best crosses for different characters showing heterosis over standard check (SC)

\begin{tabular}{|l|l|}
\hline Characters & Best Crosses \\
\hline Days to first flowering & KPMR-851 X Pant P-217 (-13.43), KPMR-851 X Prakash (-12.94) \\
\hline Number of nodes to first flowering & $\begin{array}{l}\text { Makyatmubi X Makuchabi (25.64), Makyatmubi X Prakash (25.64) } \\
\text { and Makuchabi X KPMR-851 (25.64) }\end{array}$ \\
\hline Days to 50\% flowering & $\begin{array}{l}\text { KPMR-851 X VL-58 (-11.16), Prakash X Pant P-217 (-10.70) and Pant } \\
\text { P-217 X VL-58 (-10.23). }\end{array}$ \\
\hline Days to maturity & KPMR-851 X Rachna (-4.44) and Pant P-217 X VL-58 (-4.44) \\
\hline Number of pods per plant & $\begin{array}{l}\text { Makuchabi X VL-58 (69.57) and Makyatmubi X KPMR-851 } \\
(69.57)\end{array}$ \\
\hline Pod length (cm) & $\begin{array}{l}\text { Makyatmubi X Prakash (22.05) and Makyatmubi X Pant P-217 } \\
(21.49)\end{array}$ \\
\hline Number of seeds/plant & Makuchabi X VL-58 (25.00) and Pant P-217 X Rachna (25.00) \\
\hline Seed yield/plant (g) & $\begin{array}{l}\text { Makyatmubi X KPMR-851 (192.75) and Makuchabi X VL-58 } \\
(190.42)\end{array}$ \\
\hline Biological yield/plant (g) & $\begin{array}{l}\text { Makyatmubi X KPMR-851 (135.52) and Makuchabi X VL-58 } \\
(117.14)\end{array}$ \\
\hline 100 seed weight $(\mathbf{g})$ & $\begin{array}{l}\text { Makyatmubi X Prakash (64.40) and Makyatmubi X Makuchabi } \\
(59.15)\end{array}$ \\
\hline Harvest index $(\%)$ & Makuchabi X Prakash (43.29) and Makuchabi X KPMR-851 (42.99) \\
\hline
\end{tabular}

However in majority of cases, the crosses exhibiting high sca effects were found to have either or both of the parents as good general combiner for the character under reference. Present finding is similarity with the result of Kumar et al., (2006) and Patil and Navale (2006) that most of the promising cross is the one that involves parents with high gca and shows high sca effects. The major part of such variance would be fixable in later generations. Such crosses were Makyatmbi x Prakash for 100 seed weight and pod length, Makuchabi $x$ VL-58 for seed yield/plant and Makyatmubi $x$ KPMR-851 for seed yield/plant and biological yield/plant. Recombination breeding through multiple crosses involving these hybrids would be desirable to breed genotypes having these characters. The present findings are in tune with Singh et al., (2005) and Brar et al., (2012).

In conclusion, over all it can be concluded from combining ability analysis that there is predominant role of both additive and nonadditive type of gene action for seed yield and its components. On the basis of GCA effects
Makyatmubi and Makuchabi were identified as most promising parents for involving in hybridization programme for generating desirable segregants. The manifestation of heterosis for seed yield was evidenced by superiority of hybrids ranging from 6.17 to $119.48 \%$ in the 13 crosses (Makyatmubi $\mathrm{x}$ KPMR-851, Makuchabi $x$ VL-58 and Makuchabi x Prakash, etc.) over better parent and from 42.28 to $192.75 \%$ in 19 crosses over standard check variety Rachna.

The crosses which exhibited superiority over better parent or standard parent for seed yield also exhibited significant heterosis for three to four yield components (pod length, seeds/pod, number of pods/plant and seed weight).

Further on the basis of results of mean performance, SCA effects and standard heterosis, three crosses viz., Makyatmubi $\mathrm{x}$ KPMR-851, Makuchabi xVL-58 and Makuchabi Prakash were identified as the most promising cross combinations to give transgressive segregants in later generations. 


\section{References}

Anonymous (2013). State of Indian Agriculture 2012-13. Printed and published by Directorate of Economics and Statistics, Ministry of Agriculture, Government of India. New Delhi.

Bisht, B. and Singh, Y.V. (2011). Combining ability for yield and yield contributing characters in pea. Veg. Sci., 38(1): 1721.

Borah, H. K. (2009). Studies on combining ability and heterosis in field pea. Leg. Res., 32(4): 255-259.

Brar, P.S., Dhall, R. K. and Dinesh (2012). Heterosis and combining ability in garden pea for yield and its contributing traits. Veg. Sci., 39(1): 51-54.

Esposito, M. A., Gatti, H., Cravero V.P., Anodo, F.S.L. and cointry, E.L. (2013). Combining ability and heterotic groups in Pea. Aust. J. Crop Sc., 11: 16341641.

Griffing, B. (1956). Concepts of general and specific combining ability in relation to diallel crossing system. Aust. J. Bio. Sci., 9: 463-493.

Kumar, A. and Jain, B.P. (2002). Combining ability status in pea. Indian J. Hort., 59(2): 181-184.

Kumar, S., Srivastava, R.K. and Singh, R. (2006). Combining ability for yield and its component traits in field pea. Indian J. Pulses Res., 19(2): 173-175.

Pandey, P.K., Singh, K.P. and Kar, R.M. (1996). Combining ability analysis for some quantitative characters in garden pea. Ann. Agri. Res., 17(3): 230-234.
Pant, D.C. and Bajpai, G.C. (1993). Combining ability of some leafy, semileafy and dwarf lines of field pea. Indian J. Pulses Res., 6(1): 15-20.

Patil, H.E. and Navale, P.A. (2006). Combining ability in cowpea. Legume Res., 29(4): 270-275.

Rai N and Rai M (2006) Heterosis breeding in vegetable crops.New India Publishing Agency, pp: 7-9.

Ranjan, S., Kumar, M. and Pandey, S.S. (2005). Diallel analysis for yield and yield contributing characters in pea. Legume Res., 38(3): 223-225.

Sharma, A.K., Vikas and Sharma, M.K. (2003). Studied on combining ability and gene action in pea using exotic and indigenous genotypes. Crop Res., 9(15): 201-203.

Sharma, R.N., Mishra, R.K., Pandey, R.L. and Rastogi, N.K. (1998). Study on heterosis in field pea. Ann. of Agri. Res., 19(1): 58-60.

Singh, G., Khakhar, P.S. and Srivastava, S.B.L. (2005). Combining ability in cowpea. Indian J. Pulses Res., 19(2): 25-27.

Singh, V.P., Pathak, M.M. and Singh, R.P. (1994). Combining ability in pea. Indian J. Pulses Res., 7(1): 11-14.

Srivastava, R.P. and Ali, M. (2004). Nutritional quality of common pulses. Indian Institute of Pulses Research, Kanpur, India.

Zaman, S. and Hazarika G.N. (2005). Combining ability in pea. Legume Res., 25(2): 105-108.

\section{How to cite this article:}

Manish Kumar, M.S. Jeberson, N.B. Singh and Ranjit Sharma. 2017. Genetic Analysis of Seed Yield and Its Contributing Traits and Pattern of Their Inheritance in Fieldpea (Pisum sativum L). Int.J.Curr.Microbiol.App.Sci. 6(6): 172-181. doi: https://doi.org/10.20546/ijcmas.2017.606.020 